\title{
Design and Analysis of Multilayer Solenoid Coil for Faraday Modulator
}

\author{
Muhammad Basharat ${ }^{1, a}$, Ming Ding ${ }^{1}$, Hongwei Cai ${ }^{1}$, Yang $\mathrm{Li}^{1}$ and Jiancheng Fang ${ }^{1}$ \\ School of Instrumentation Science \& Opto-Electronics Engineering, Beihang University, Beijing 100191, PR China
}

\begin{abstract}
A well-designed polarimeter is integral to the realization of the highly sensitive atomic magnetometer. Amongst various detection schemes, optical polarimetry based on Faraday modulator is most commonly used owing to its angular sensitivity at low-frequency range. The multilayered solenoid coil is the key component of Faraday effect based optical detection system. This paper primarily deals with design and analysis of multilayered coil of Faraday modulator. All deterministic parameters that affect the optimum design have been identified and analyzed. Mathematical expressions have been obtained for axial field homogeneity; modulation depth and power dissipation manifesting direct dependence on coil geometry. The design parameters of the coil are optimized with respect to axial magnetic field homogeneity over region of interest and reduced power losses with suitable geometric construction. The influence of different geometrical and electromagnetic parameters on optimum design has been highlighted and guidelines for design procedure are given. Theoretical results have been compared with simulation and experimental results. The mathematical formulation could be implemented in a computer program for recurrence design and to assist the realization of an optimized design of Faraday modulator coil.
\end{abstract}

\section{Introduction}

Various scientific and technological applications demand homogeneous alternating magnetic field generated by solenoid coils [1-4]. Typical applications include optical magentometery [5], spectroscopy [6] and biomedical applications [7-9]. Air-core solenoid coils are preferred choice owing to high peak amplitude field and an internal cavity for holding the test element to be magnetically permeated. However, in general, the magnetic field generated by simple solenoid coils is uniform over the limited region. The magnetic field of multilayered solenoid coils is strongly geometry dependent. The optimization of these coils is not merely maximizing the magnetic field over a region of interest rather other associated attributes have to be considered as well. Several coil geometries have been reported for field shaping [10]. The axial field homogeneity can be achieved by increasing the number of turns but it reduces the amplitude of the field for given excitation voltage [11]. On the other hand, poor axial field homogeneity is exhibited by shorter coils having fewer turns. In this case, the span of the peak field is over a limited region and decreases rapidly along the axis of the coil. In general, the dimensions of the coils are larger than the volume of interest. There is always a need for reducing the dimensions without compromising uniformity of field.

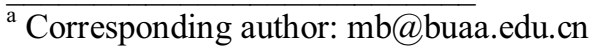


Sensitive optical magnetometer requires the detection of the extremely small rotation angle. Among various detection schemes, optical polarimetry based on Faraday modulator is most commonly used. The modulation depth of Faraday modulator depends on upon the Verdet constant of the optical material and magnetic field of the multilayered solenoid coil. The optimized performance of modulator depends on upon various parameters. Paramagnetic optical glasses having high Verdet constants are used for large modulation depth. The Verdet constant of paramagnetic glasses is strongly temperature dependent. This dependence dictates the requirement of no heat generated by coil during excitation of the coil. For the optimized performance of the modulator, a tradeoff of different working parameters of coils is inevitable.

The purpose of this work is twofold. First, it aims to identify and analyze all potential geometric and electromagnetic parameters affect the design of the Faraday modulator coil. Second, to develop a mathematical formulation to obtain an optimum design of the coil with the following requirement: (a) Uniform axial magnetic field over the region of interest with suitable geomantic construction and (b) maximize the modulation depth of modulator at the expense of reduced power losses of the coil. The developed formulation could be implemented easily in a computer program for recurrence design. This work is not only limited to the design of Faraday modulator coil but also equally useful for the designing of the coils required for precision instrumentation based on magneto-optics.

\section{Design parameters analysis}

In this section various geometrical and electromagnetic parameters require for initiation of design are listed and their characteristics effect on the optimum design of multilayered solenoid coil has been analyzed. The interdependence of these parameters has been established. A multi-layered solenoid coil refers to a system many layers of magnetic wire wound on a suitable former. The basic structure of multilayered solenoid coil is shown in Figure 1. Where $L$ is length, $r_{1}$ is internal radius; $r_{2}$ is the outer radius of the coil, while $d$ is magnetic wire diameter.

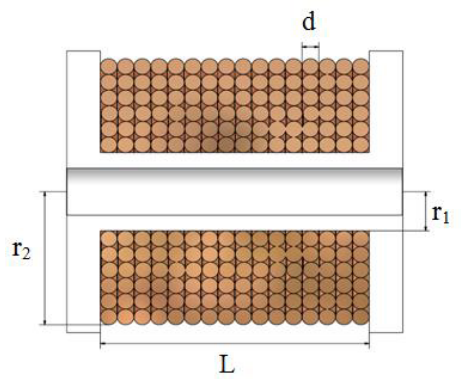

(a) (b)

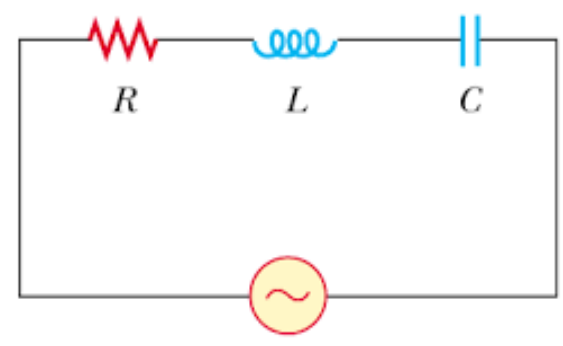

Figure 1. (a) The geometry of the coil, (b) and its equivalent magnetic circuit.

\subsection{Geometric and electrical parameters}

The number of turns of coil, which depends on diameter $d$ of magnetic wire, coil packing factor $k$, and dimension of the coil, are given by

$$
n=\frac{l\left(r_{2}-r_{1}\right)}{k d^{2}}
$$

The coil packing factor $k$ is the ratio of total conduction area of the winding and coil cross section window area. The packing factor may range from $0.2-0.9$. With bobbin core, $k$ may be as high as 0.8 for a tight winding.

The approximate length of wire needed for winding can be computed for by the relation 


$$
l_{w}=\pi \frac{4\left(r_{2}^{2}-r_{1}^{2}\right)}{d^{2}} l
$$

The area of the cross-section magnetic wire is given by:

$$
A_{w}=\frac{\pi d^{2}}{4}
$$

The ohmic resistance of the coil is given by

$$
R_{c}=\frac{l_{w}}{A_{w} \sigma_{c u}}
$$

Where $\sigma_{c u}$ is resistivity of copper.

The inductance of multilayered solenoid coil can be computed by the relation [12]

$$
L=\frac{78.7 n^{2} d_{m}^{2}}{3 d_{m}+5\left(d_{2}-d_{1}\right)+9 l}
$$

Where $d_{m}$ is mean diameter of the coil.

The impedance of coil is given by

$$
Z_{c}=\omega L
$$

The coil is resonated by impedance matching; the value of capacitance required is computed by the relation

$$
C=\frac{1}{\omega Z_{c}}
$$

If the circuit is properly tuned, the resonant voltage developed across capacitor and coil

$$
V_{c}=A b s\left[V\left(\frac{\omega l}{R_{e f f}}+1\right)\right]
$$

\subsection{Copper losses}

The DC resistance of the coil depends on dimensions of the winding, the number of turns and the material of magnetic wire. This DC resistance induces the copper loss $P_{c u}$, it is given by

$$
P_{c u}=\rho_{c u} \frac{l_{w}}{A_{w}} I_{r m s}
$$

Where $\rho_{\mathrm{cu}}$ is resistivity of the copper $l_{w}$ is the length of the magnetic wire.

\subsection{Eddy losses}

In the case of $\mathrm{AC}$, a magnetic flux is produced around the conductor and an eddy current is induced by these flux lines. This eddy current flows in such way that it opposes the induced magnetic flux. The induced eddy current flows in the interior of the conductor to a direction opposite to applied AC current. Consequently, the interior of the conductor is shielded from the applied current. The current density inside the conductor reduces. The depth of the conductor at which internal current density becomes $1 / e$ of the surface current density is termed as skin depth. The skin depth of magnetic wire at given excitation frequency is computed as: 


$$
\delta=\sqrt{\frac{2}{\mu_{0} \sigma_{c u} \omega}}, \omega=2 \pi f
$$

Where, $\mu_{o}$ is permittivity of free space, $\sigma_{c u}$ electrical conductivity of copper and $f$ is the excitation current frequency. The effective conduction area of conductor reduces which cause an increase in the $\mathrm{AC}$ resistance and additional resistive losses.

Another dominant factor which causes an increase in resistance is proximity effect. The current in a first conductor is constrained to smaller region due to the proximity of another conductor. The model developed by Dowell [13] includes both effects and modify the DC resistance accordingly. In this method, round wire is approximated as equivalent foil rectangle wires. For a multilayer coil, the $\mathrm{AC}$ resistance is expressed as

$$
R_{A C}=\left(\Delta\left[G_{1}(\Delta)+\frac{2}{3}\left(n_{l}^{2}-1\right)\left(G_{1}(\Delta)+G_{2}(\Delta)\right)\right]\right) R_{D C}
$$

Where $n_{l}$ is number of layer in coil, $\Delta$ correction factor due to replacement of rectangular wire

$$
\Delta=\frac{d}{\delta} \sqrt{\frac{\pi}{2}}
$$

$G_{1}$ and $G_{2}$ represents the geometry of the infinite coil

$$
\begin{gathered}
G_{1}(\Delta)=\frac{\sinh (2 \Delta)+\sin (2 \Delta)}{\cosh (2 \Delta)-\cos (2 \Delta)} \\
G_{2}(\Delta)=\frac{\sinh (\Delta) \cos (\Delta)+\sin (\Delta) \cosh (2 \Delta)}{\cosh (2 \Delta)-\cos (2 \Delta)}
\end{gathered}
$$
[14]:

The effective resistance $R_{\text {eff }}=R_{A C}$ for sinusoidal excitation can be approximated by the relation

$$
R_{\text {eff }}=R_{D C}\left(1+\frac{\psi}{3} \Delta^{4}\right)
$$

Where, $\psi=\frac{5 n_{l}-1}{15}, n_{l}$ is the number of layers in the coil.

\subsection{Magnetic field}

In order to express the magnetic field of the coil in terms of its geometrical parameters, it instructive to use rectangular coordinates. The magnetic field intensity $B(z)$ due to the finite multilayered coil at the point $z$ on the axis is [15].

$$
B(z)=\frac{\mu_{0} n i}{2 L\left(r_{2}-r_{1}\right)}\left[A \ln \frac{r_{2}+\sqrt{r_{2}^{2}+A^{2}}}{r_{1}+\sqrt{r_{1}^{2}+A^{2}}}+B \ln \frac{r_{2}+\sqrt{r_{2}^{2}+B^{2}}}{r_{1}+\sqrt{r_{1}^{2}+B^{2}}}\right]
$$

Where $A=L / 2-z, B=L / 2+z$, while $L, r_{1}$ and $r_{2}$ are the length, inner radius, and outer radius of the coil respectively. Expressing the axial magnetic field in this form allows the mapping of the field distribution along the axis by knowing the dimensions of the coil.

\section{Results and discussion}




\subsection{Magnetic field homogeneity}

The variability of the magnetic field within the volume of interest can be measured and expressed in the form of magnetic field homogeneity $(H)$. Normally this variation is expressed as a deviation of magnetic fields from the peak field at the center and it is given by

$$
H=\frac{B_{0}-B_{z}}{B_{0}}
$$

The extent of magnetic field homogeneity is application dependent. The dependence of parameter $H$ on the dimensions of the coil can be worked out from Eqs. (16) and (17)

$$
H=1-\frac{\left[(L / 2-z) \ln \frac{r_{2}+\sqrt{r_{2}^{2}+(L / 2-z)^{2}}}{r_{1}+\sqrt{r_{1}^{2}+(L / 2-z)^{2}}}+(L / 2+z) \ln \frac{r_{2}+\sqrt{r_{2}^{2}+(L / 2+z)^{2}}}{r_{1}+\sqrt{r_{1}^{2}+(L / 2+z)^{2}}}\right]}{L \ln \frac{r_{2}+\sqrt{r_{2}^{2}+(L / 2)^{2}}}{r_{1}+\sqrt{r_{1}^{2}+(L / 2)^{2}}}}
$$

The Eq.(18) shows that magnetic field homogeneity depends upon coil geometry only.

Figure 2(a) shows the variation of the axial magnetic field of a coil having inner diameter $15 \mathrm{~cm}$, outer diameter $35 \mathrm{~mm}$ and length of $100 \mathrm{~mm}$ at $3 \mathrm{~A}$ current. The homogeneity of axial field is shown in Figure 2(b). Theoretical results have been compared with experimental results in reported in [16]. There is good agreement between experimental and theoretical results over the central region of the curves. At the edges the experimental curve deviates slightly from the theoretical one, it is due to the fact the magnetic field profile of a real coil is prone to errors due to the winding geometry, direction of the windings, errors in wire cross-sections and the insulation layer, filling factor, the geometry of the coil former etc. This result proves the usefulness of the expression given in Eq.(18) for calculating the homogeneity of the magnetic field profile by knowing only the dimensions of the coil with fair accuracy.
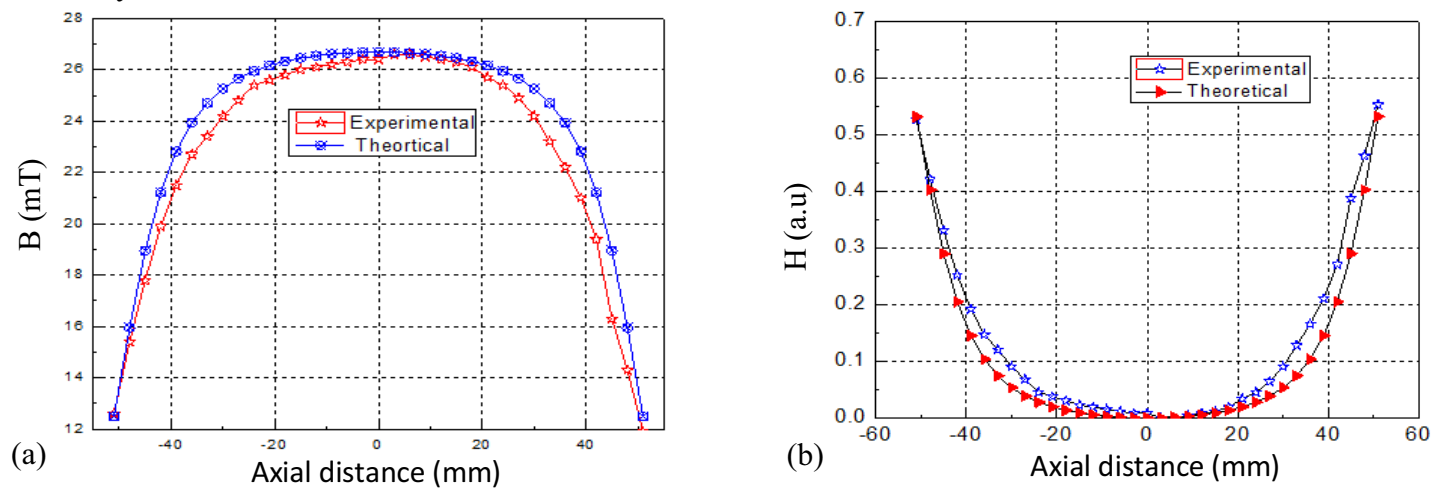

Figure 2. (a) Axial magnetic field variation as a function of distance on the axis of the coil, (b) Homogeneity of the axial magnetic field.

To evaluate the dependence of homogeneity of the coil on its dimensions, the normalized magnetic field distribution curves have been obtained as a function of coil dimension ratios namely length to inner diameter $(\alpha)$ and outer to the inner diameter $(\beta)$. Figure 3(a) shows regions with a uniform magnetic field for different values of $\alpha$ for fixed value of $\beta$. For large values of $\alpha$, the homogeneity region increases appreciably. As in this case, the inner diameter has been held constant the large value $\alpha$ implies the larger length of the coil. This shows that homogeneity region increases with increasing the length of the coil. For coil dimension $\alpha \geq 10 \beta$ the homogeneity region with $10 \%$ variation extends to about $80 \%$ of the coil length while half of the coil length has homogeneity with variation up to $1 \%$. The coil whose magnetic field profile is given in Figure 2 has dimension $\alpha=5.78 \beta$ and it has a close correspondence with the curve corresponding to $\alpha=10$ in Figure 3(a). 
The analysis of axial field homogeneity distribution has been performed using COMSOL Multiphysics. The simulation results of two representative coil configurations are shown in Figure 3(b). Longer coil has parameters $\mathrm{L}=100 \mathrm{~m}, \mathrm{r}_{1}=7.5 \mathrm{~mm}, \mathrm{r}_{2}=17.5$, and $\mathrm{n}=730$, while shorter has parameters $\mathrm{L}=70 \mathrm{~m}, \mathrm{r}_{1}=24.5 \mathrm{~mm}, \mathrm{r}_{2}=30.5$, and $\mathrm{n}=360$. Both coils are excited at $3 \mathrm{~A}$ current. It is evident that longer coil has uniform magnetic field distribution over extended region compared to the shorter coil.

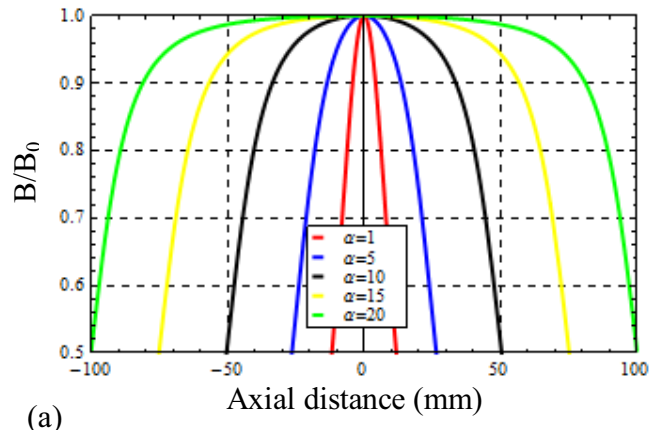

(b)
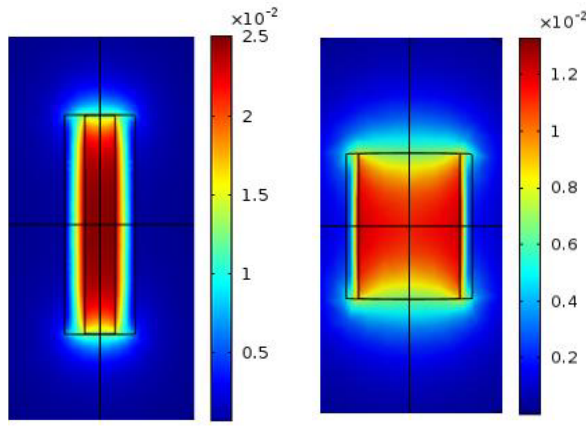

Figure 3. (a) Normalized axial magnetic field for different values of $\alpha\left(L / r_{1}\right)$ at $\beta\left(r_{2} / r_{1}\right)=2$, (b) Multiphysics simulation of magnetic field distribution (measured in Tesla) of two representative coil conFigureurations.

In Figure 4(a), the normalized magnetic field has been plotted as a function of $\boldsymbol{\alpha}$ for constant coil length and $\beta$. It was observed that in this case, inner diameter has characteristics effect on the homogeneity of the magnetic field. For large values of $\alpha$ i.e. smaller inner diameter, the homogeneity regions extend concluding that smallest possible values of inner diameter are desirable. This fact has accentuated by simulation results shown in Figure 3(b). Normalized magnetic field of the square coils $\left(\mathrm{L}=2 \mathrm{r}_{2}\right)$ for different values of $\beta$ is shown in Figure 4(b). In this case length and outer diameter have been kept constant while inner diameter has been varied. It is shown that the square coils have poor axial field homogeneity.

(a)

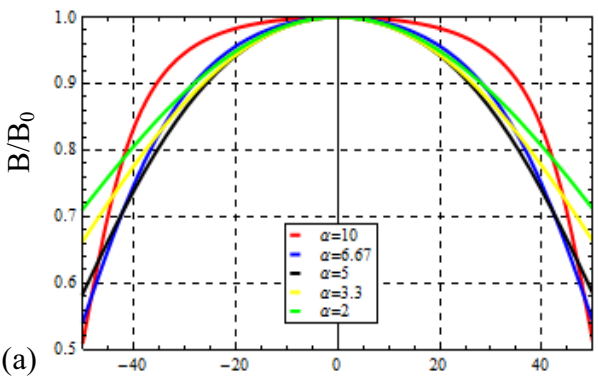

Axial distance $(\mathrm{mm})$

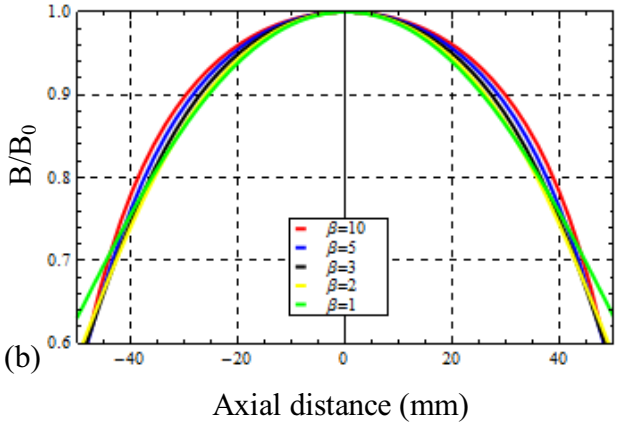

Axial distance (mm)

Figure 4. (a) Normalized axial magnetic field for different values of $\alpha\left(L / r_{1}\right)$ at fixed length of the coil., (b) Normalized axial magnetic field for different values of $\alpha\left(\mathrm{L} / \mathrm{r}_{1}\right)$ for square coils.

\subsection{Modulation depth and power dissipation}

When solenoid coil is assembled in Faraday modulator, an optical glass is placed inside of bore of air core multilayered solenoid coil. The rotation of the plane of polarization of a plane due to the axial magnetic field of coil is given by

$$
\theta=V \int_{-L}^{L} B(z) d z
$$


Where $\theta$ is the angle of rotation (modulation depth), $V$ is Verdet constant of the optical glass of length $L$, and $B(z)$ is applied axial magnetic field density.

From Eqs. (16) and (19)

$$
\begin{aligned}
& \theta=\frac{\mu_{0} n i V}{2 L\left(r_{2}-r_{1}\right)}\left[\left(L^{2} \ln \frac{r_{2}+\sqrt{r_{2}^{2}+L^{2}}}{r_{1}+\sqrt{r_{1}^{2}+L^{2}}}+r_{2} \sqrt{r_{2}^{2}+L^{2}}-r_{1} \sqrt{r_{1}^{2}+L^{2}}\right)\right. \\
& \left.-\left(\frac{L^{2}}{4} \ln \frac{r_{2}+\sqrt{r_{2}^{2}+L^{2} / 4}}{r_{1}+\sqrt{r_{1}^{2}+L^{2} / 4}}+r_{2} \sqrt{r_{2}^{2}+L^{2} / 4}-r_{1} \sqrt{r_{1}^{2}+L^{2} / 4}\right)\right]
\end{aligned}
$$

Eq. (20) can be rearranged

$$
\begin{aligned}
& \chi=\frac{\theta}{n i}=\frac{K}{\alpha(\beta-1)}\left[\left(\alpha^{2} \ln \beta \frac{1+\sqrt{1+A^{2}}}{1+\sqrt{1+\alpha^{2}}}+\beta^{2} \sqrt{1+A^{2}}\right.\right. \\
& \left.\left.-\sqrt{1+\alpha^{2}}\right)-\left(\frac{\alpha^{2}}{4} \ln \beta \frac{1+\sqrt{1+A^{2} / 4}}{1+\sqrt{1+\alpha^{2} / 4}}+\beta^{2} \sqrt{1+A^{2} / 4}-\sqrt{1+\alpha^{2} / 4}\right)\right]
\end{aligned}
$$

Where $\alpha=L / r_{1}, \quad A=L / r_{2}, \quad \beta=r_{2} / r_{1}, \quad K=\frac{\mu_{0} V}{2}$ is a constant, and $\chi=\frac{\theta}{n i}$ is figure merit of the Faraday modulator relating rotation to ampere-turn of the coil

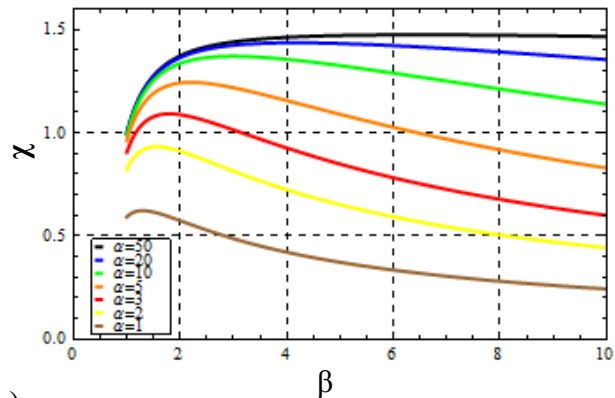

(a)

Figure 5. (a) The ratio of rotation to ampere-turn as a function of coil dimension ratios $\alpha$ and $\beta$, (b) 3D graph showing the ratio of rotation to ampere-turn as a function of coil dimension ratios $\alpha$ and $\beta$ for the whole range.

The plot of $\chi$ vs coil radii ratio $\beta\left(\mathrm{r}_{2} / \mathrm{r}_{1}\right)$ is shown in Figure 5. It can be seen that $\chi$ is dependent only on the ratios of coil dimensions and its values are large for the thin coils. However, we have to consider the power needed for required modulation depth.

The power dissipated in coil excited by alternating current is given by

$$
P=i^{2} R_{e f f}
$$

If optimal diameter conductor is used for winding, the $R_{\text {eff }}$ of multilayer coil using round conductor is two times of $R_{D C}$ [17].

$$
P=2 I^{2} R_{D C}
$$

From Eqs. (1), (2), (4) and using the fact $k=\frac{n A_{c u}}{L\left(r_{2}-r_{1}\right)}$

$$
P=\frac{8 i^{2} \rho \pi\left(r_{1}+r_{2}\right) n^{2}}{L\left(r_{2}-r_{1}\right)}
$$




$$
\frac{n i}{\sqrt{P}}=\sqrt{\frac{L(\beta-1)}{8 \rho \pi(\beta+1)}}
$$

Where $\beta=r_{2} / r_{1}$

From Eqs. (21) and (25)

$$
\zeta=\frac{\theta}{\sqrt{P}}=k \chi \sqrt{\frac{(\beta-1)}{(\beta+1)}}
$$

Where $k$ is constant and $\zeta=\frac{\theta}{\sqrt{P}}$ is figure merit of the Faraday modulator relating rotation to square of power dissipation of the coil

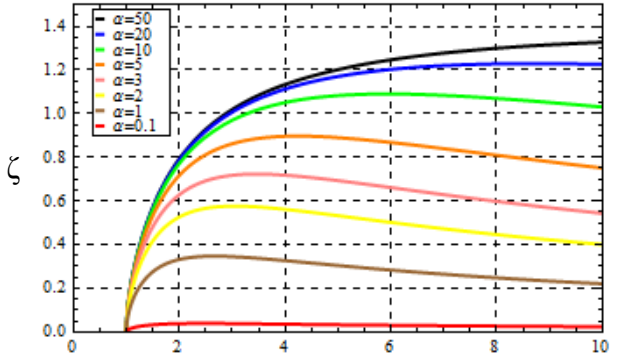

(a)

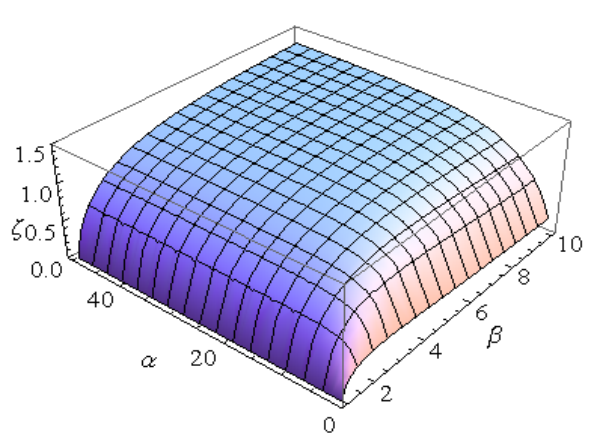

(b)

Figure 6. (a) The ratio of the rotation to $\sqrt{\mathrm{P}}$ as a function of coil dimension ratios $\alpha$ and $\beta$, (b) 3D graph showing the ratio of rotation to $\sqrt{\mathrm{P}}$ as a function of coil dimension ratios $\alpha$ and $\beta$ for the whole range.

Figure 6 shows that there are well-defined values of the coil radii ratio $\beta\left(\mathrm{r}_{2} / \mathrm{r}_{1}\right)$ corresponding to each value of $\alpha$ which maximize $\zeta$. The power needed for a required rotation for a given coil dimensions depend upon the ampere-turns rather than separately on the current or on the coil turns.

The results of this section indicate that the magnetic field homogeneity, modulation depth and power dissipation of the coil are geometry dependent and can be expressed in terms coil dimension ratios. The mathematical formulation given in section 2 and 3 could be implemented in a computer program easily. For instance, we have implemented this into Matlab GUI to assist the design of the coil. Nevertheless, the analysis and findings presented here can be used to design a solenoid coil without any computer program as well. Before proceeding one has to decide what can be changed and what is fixed. In most of the cases, the internal diameter of the coil cannot be changed at will as the optical material along with holding mechanism must be fitted snugly inside it. Based on geometric constraints of the experiment, initial values of length and the inner diameter of the required coil are established. An appropriate gauge of magnetic wire is selected to meet the requirement of frequency and operating voltage and current rating. Depending upon the level of homogeneity of the axial magnetic field and modulation depth for a particular experiment the dimensions of the coil for optimum design are found following the results presented in section 3. Once the design parameters are finalized the working parameters are calculated from the analysis given section 2. By doing impedance matching the resonant rise in voltage may be up to hundreds of Volts, therefore, proper insulation of the coil terminals should be insured.

\section{Conclusion}

The optimum design of multilayer solenoid coil implies a trade-off between the technical complicacy and best performance parameter such homogeneity, modulation depth and so on. The parameters that affect the optimal design have been identified and analyzed. The interdependence of these parameters 
has been formulated. The optimum design has been obtained with respect axial magnetic field homogeneity over a region of interest and optimum modulation depth with reduced power losses with suitable geometric construction. The results obtained from this investigation indicate the performance parameters of the coil are geometry dependent and can be manipulated in terms coil dimension ratios. The developed formulation could be implemented in a computer program easily for estimating the optimality of the coil design. This work is not limited only to the design of Faraday modulator coil but also equally useful for the designing of the coils required for precision instrumentation based on magneto-optics.

\section{References}

1. N. K. Das, P. Barat, S. Dey, and T. Jayakumar, Prog. Electromagn. Res. M 34, 99 (2014)

2. S. Sato, S. Sakaguchi, K. Futamata, and K. Katou, IEEE Trans. Magn. 36, 649 (2000)

3. J. L. Kirschvink, Bioelectromagnetics 13, 401 (1992).

4. R. Merritt, C. Purcell, and G. Stroink, Rev. Sci. Instrum. 54, No. 879(1983)

5. S. J. Seltzer, "Developments in alkali-metal atomic magentometery," Ph.D. dissertation (Princeton University, 2008).

6. K. Ehrmann, N. Saillen, F. Vincent, M. Stettler, M. Jordan, F. M. Wurm, P.A. Besse, and R. Popovica, Lab Chip. 7, 373 (2007)

7. K. R. Minard and R. A. Wind, Concepts Magn.Reson. 13, 128 (2000)

8. C. McCann and M. D. Sherar, Phys. Med. Biol. 51, 3851 (2006)

9. D.E. Bordelon, R.C. Goldstein, V. S. Nemkov, A.Kumar, John K. Jackowski, T.L. DeWeese, and R.Ivkov IEEE Trans. Magn. 48, 47 (2012)

10. P. R. Stauffer, P. K. Sneed, H. Hashemi, and T. L. Phillips, IEEE Trans. Biomed. Eng. 41, 17 (1994)

11. T. Tominaka, Eur. J. Phys. 27, 1399 (2006)

12. P.Ripka, Magnetic Sensors and Magnetometer (Artech House Inc, 2001)

13. P. L. Dowell, Proc. Inst. Electr. Eng. 113, 1387 (1966)

14. W.G. Hurley, E. Gath, J.G. Breslin, IEEE Trans. Power Electron. 15, 369, (2000)

15. R. M. Duffy and R. P. Netterfield, Rev. Sci. Instrum. 55, 743, (1984)

16. M. Basharat, Z. Razzaq, S. Javed, and U. Afzal, OAM-RC. 8, 930 (2014)

17. R.P. Wojda Marian, K. Kazimierczuk, COMPEL - The international journal for computation and mathematics in electrical and electronic engineering, 31, 1793(2012) 